\title{
MODELAGEM DOS SISTEMAS DE ADMISSÃO DE AR PARA MOTORES COM TECNOLOGIA FLEX
}

\author{
Marcos Henrique C. Silva ${ }^{1}$, Armando Antônio M. Laganá \\ ${ }^{1,2}$ Escola Politécnica da Universidade de São Paulo \\ ${ }^{2}$ Fatec Santo André
}

E-mails: marcoshencarsil@gmail.com, armandolagana@terra.com.br

\section{RESUMO}

Neste artigo é apresentado um modelo do sistema de admissão de ar para motores com tecnologia FLEX. O objetivo é fornecer uma formulação matemática para, por meio de análise teórica e métodos de identificação experimental, poder predizer a massa gasosa admitida pelo cilindro através do sinal dos atuadores e sensores deste sistema. Para o caso dos motores convencionais, esses atuadores são a válvula borboleta e a válvula de recirculação, e o sensoriamento é operado apenas pelo MAP. Para formular esta relação, estudos sobre o comportamento dos fenômenos nas válvulas, nos coletores, nos dutos e no próprio cilindro são desenvolvidos. Análises específicas sobre como a composição influencia estes fenômenos são realizadas, de forma a poder empregar estes modelos em motores com tecnologia FLEX. Métodos de identificação experimental para o caso específico da modelagem de sistema de admissão são apresentados, visando aproximar os resultados experimentais das formulações do modelo.

\section{INTRODUÇÃO}

O sistema de admissão de ar pode ser dividido nos seguintes subsistemas: válvulas, coletores e cilindro. Neste artigo, será feita a modelagem de cada um destes subsistemas de forma a predizer a massa de ar admitida, grandeza necessária para fazer o design e execução de controles, como o do torque e da relação ar/combustível. 
Todas as variáveis que sofrem modificação no uso de composição variada gasolina/etanol foram abordadas e discutidas, para que se entenda como estes modelos podem ser empregados para motores com tecnologia FLEX.

\section{Modelagem de coletores}

Ao modelar coletores, pode-se ter interesse em vários aspectos. Neste artigo estaremos interessados em modelar os fluxos através de um coletor, a temperatura dos gases reservados no coletor e a pressão interna.

Assumiremos que o coletor é um sistema de parâmetros agrupados, que não há transferência de massa ou calor através das paredes e que não há variação substancial da energia cinética e potencial dos fluxos no coletor. Este será modelado como um reservatório de energia e massa, no qual adentra e sai fluxos mássicos e energéticos. Portanto, a diferença entre os fluxos mássicos de entrada e saída corresponde à derivada temporal da massa reservada e a diferença entre os fluxos energéticos de entrada e saída corresponde à derivada temporal da energia reservada.

Usa-se formulação adiabática quando o fluxo gasoso atravessa brevemente o coletor e quando a razão área/volume do coletor é pequena. Segue abaixo esta formulação [1]:

$$
\begin{gathered}
\frac{d}{d t} p(t)=\frac{\kappa \cdot R}{V} \cdot\left[\dot{m}_{E}(t) \cdot \vartheta_{E}(t)-\dot{m}_{S}(t) \cdot \vartheta_{S}(t)\right] \\
\frac{d}{d t} \vartheta_{S}=\frac{\vartheta_{S} \cdot R}{p \cdot V \cdot c_{v}} \cdot\left[c_{p} \cdot \dot{m}_{E} \cdot \vartheta_{E}-c_{p} \cdot \dot{m_{S}} \cdot \vartheta_{S}-c_{v} \cdot\left(\dot{m}_{E}-\dot{m_{S}}\right) \cdot \vartheta_{S}\right]
\end{gathered}
$$

Aonde,

$p(t)$ : Pressão no coletor; $\kappa:$ Razão dos calores específicos, ou seja, $\kappa=\frac{c_{p}}{c_{v}} ; R$ : Constante universal dos gases; $V$ : Volume do coletor; $\dot{m}_{E}$ : Fluxo mássico de entrada; $\vartheta_{E}$ : Temperatura dos gases no fluxo de entrada; $\dot{m}_{S}$ : Fluxo mássico de saída; $\vartheta_{S}$ : 
Temperatura dos gases no fluxo de saída; $c_{p}$ : Capacidade calorífica dos gases a pressão constante; $c_{\mathrm{v}}$ : Capacidade calorífica dos gases a volume constante.

Caso ocorra o inverso (fluxo demora para atravessar o coletor ou razão área/volume é considerável), podemos usar a formulação isotérmica abaixo [1]:

$$
\begin{gathered}
\frac{d}{d t} p(t)=\frac{R \cdot \vartheta(t)}{V}\left[\dot{m_{E}}-\dot{m}_{S}\right] \\
\vartheta(t)=\vartheta_{E}(t)
\end{gathered}
$$

A capacidade calorífica específica molar à pressão constante do ar admitido, dos gases queimados da combustão completa e estequiométrica do etanol e dos gases queimados da combustão completa e estequiométrica da gasolina são na temperatura ambiente, respectivamente, $C_{p}=3,51 \cdot R, C_{p}=3,67 \cdot R$ e $C_{p}=3,72 \cdot R$. A massa molar média destas misturas são, respectivamente, $M=28,8 \mathrm{~g} / \mathrm{mol}, M=$ $28,9 \mathrm{~g} / \mathrm{mol}$ e $M=28,7 \mathrm{~g} / \mathrm{mol}$. Como estamos considerando gases recirculados refrigerados, vamos desprezar as diferenças de temperatura entre o fluxo de gás recirculado e o ar admitido, devido à diferença destas duas temperaturas ser insuficiente para provocar mudanças significativas nos valores da capacidade calorífica [2]. Logo, pelos valores acima, podemos aproximar os valores de capacidade calorífica molar específica e massa molar média dos três fluxos por $C_{p}=$ $3,60 \cdot R$ e $M=28,8 \mathrm{~g} / \mathrm{mol}$. Portanto, para o caso das equações (1) a (4), infere-se que estes três fluxos podem ser aproximados como de mesma natureza.

Repare que estamos desconsiderando combustão incompleta, excesso de oxigênio ou vapores de combustível nos gases recirculados. A presença de produtos de combustão incompleta ou excesso de oxigênio altera insignificantemente os valores de $C_{p}$ e $M$. Entretanto, os vapores de combustível influem significativamente nestes fatores. Entretanto, como deve-se gastar pouco tempo nos pontos de operação longe da estequiometria, pode-se desprezar a existência de tais vapores nos gases recirculados. 
No caso de identificar o fluxo através de um único cilindro, é suficiente integrar o fluxo de massa de saída do coletor de admissão (ou o fluxo de massa de entrada no coletor de escape) e dividir pelo número de cilindros para se ter o valor do fluxo por cilindro.

\section{Modelagem de válvulas}

A válvula é modelada como uma placa plana ligada ao duto através de um conector central esférico com diâmetro $d$ [3]. A abertura da válvula é modelada como um orifício [1] por onde o fluxo, vindo de um duto com área $A_{D U T O}$ e diâmetro $D$, passa através de um orifício com área $A(\theta)$, que corresponde à área de abertura da válvula. Segue abaixo o equacionamento de $A(\theta)$ [3]:

$$
\begin{gathered}
A(\theta)=\frac{-d \cdot D}{2} \cdot\left[1-\left(\frac{d}{D}\right)^{2}\right]^{\frac{1}{2}}+\frac{d \cdot D}{2} \cdot\left[1-\left(\frac{d \cdot \cos \left(\theta_{0}\right)}{D \cdot \cos \left(\theta_{0}+\theta\right)}\right)^{2}\right]^{\frac{1}{2}}+\frac{D^{2}}{2} \\
\cdot \arcsin \left(\left[1-\left(\frac{d}{D}\right)^{2}\right]^{\frac{1}{2}}\right)-\frac{D^{2}}{2} \cdot\left(\frac{\cos \left(\theta_{0}+\theta\right)}{\cos \left(\theta_{0}\right)}\right) \\
\cdot \arcsin \left(\left[1-\left(\frac{d \cdot \cos \left(\theta_{0}\right)}{D \cdot \cos \left(\theta_{0}+\theta\right)}\right)^{2}\right]^{\frac{1}{2}}\right)
\end{gathered}
$$

Aonde,

$A(\theta)$ : Área de abertura da válvula em função do ângulo de abertura da válvula; $d$ : Diâmetro do conector da válvula; $D$ : Diâmetro do duto no qual situa-se a válvula; $\theta_{0}$ : Ângulo da válvula na posição em que ela se encontra fechada; $\theta$ : Ângulo de abertura da válvula com relação à válvula fechada, ou seja, quando a válvula estiver fechada, $\theta=0$.

Para ângulos elevados de abertura, temos que a área de abertura da válvula passa a ser apenas função do diâmetro do duto e do diâmetro do conector da válvula, conforme abaixo [4]: 


$$
\begin{gathered}
A(\theta)=\frac{D^{2}}{2} \cdot \arcsin \left(\left[1-\left(\frac{d}{D}\right)^{2}\right]^{\frac{1}{2}}\right)-\frac{d \cdot D}{2} \cdot\left[1-\left(\frac{d}{D}\right)^{2}\right]^{\frac{1}{2}} \\
\text { para } \theta \geq\left(\arccos \left(\frac{d}{D} \cdot \cos \left(\theta_{0}\right)\right)-\theta_{0}\right)
\end{gathered}
$$

Quando a válvula está fechada ainda há fluxo de ar devido a tolerâncias de manufatura. Logo, para este caso [4]:

$$
\theta_{0}=0.91 \cdot \widehat{\theta_{0}}-2.59
$$

Aonde,

$\theta_{0}$ : Valor a ser considerado quando $\theta \approx 0$; $\widehat{\theta_{0}}$ : Valor verdadeiro do ângulo de fechamento da válvula.

O fluxo que passa através de uma válvula, desprezando perdas energéticas (modelagem isentálpica), é formulado como [3]:

$$
\dot{m}(t)=\frac{c_{d} \cdot A(t) \cdot p_{E}(t)}{\sqrt{R \cdot \vartheta_{E}(t)}} \cdot \Psi\left(\frac{p_{E}(t)}{p_{S}(t)}\right)
$$

Aonde,

$\dot{m}(t)$ : Fluxo de massa através da válvula; $c_{d}$ : Coeficiente de descarga da válvula; $A(t)$ : Área de abertura da válvula; $p_{E}(t)$ : Pressão a montante da válvula; $p_{S}(t)$ : Pressão a jusante da válvula; $R$ : Constante universal dos gases; $\vartheta_{E}(t)$ : Temperatura a montante da válvula; $\Psi($.$) : Função fluxo.$

A função fluxo é definida como [1]:

$$
\Psi\left(\frac{p_{E}(t)}{p_{S}(t)}\right)= \begin{cases}\sqrt{\kappa \cdot\left[\frac{2}{\kappa+1}\right]^{\frac{\kappa+1}{\kappa-1}}} & \text { para } p_{S}<p_{C R} \\ {\left[\frac{p_{S}(t)}{p_{E}(t)}\right]^{\frac{1}{\kappa}} \cdot \sqrt{\frac{2 \cdot \kappa}{\kappa-1} \cdot\left[1-\left(\frac{p_{S}(t)}{p_{E}(t)}\right)^{\frac{\kappa-1}{\kappa}}\right]}} & \text { para } p_{S} \geq p_{C R}\end{cases}
$$




$$
p_{c r}=\left[\frac{2}{\kappa+1}\right]^{\frac{\kappa}{\kappa-1}} \cdot p_{E}
$$

Aonde,

$\kappa:$ Razão dos calores específicos, ou seja, $\kappa=\frac{c_{p}}{c_{v}} ; p_{c r}$ : Pressão crítica aonde o fluxo atinge condições sônicas no orifício.

Para fluxos próximos à temperatura ambiente (ou seja, com $\kappa \approx 1.4$ ), podemos simplificar a função fluxo para [1]:

$$
\Psi\left(\frac{p_{E}(t)}{p_{S}(t)}\right) \approx \begin{cases}\frac{1}{\sqrt{2}} & \text { para } p_{S}<\frac{1}{2} \cdot p_{E} \\ \sqrt{\frac{2 \cdot p_{S}}{p_{E}} \cdot\left[1-\frac{p_{S}}{p_{E}}\right]} & \text { para } p_{S} \geq \frac{1}{2} \cdot p_{E}\end{cases}
$$

Estudos realizados em [4] mostram que o coeficiente de descarga varia conforme o ângulo de abertura da válvula. Logo, criaremos uma função $\operatorname{CV}(\theta)$ de forma a expressar num único termo a dependência com o ângulo e, a seguir, normalizaremos esta função em $F_{\theta}(\theta)$, conforme abaixo:

$$
\begin{gathered}
C V(\theta)=c_{d}(\theta) \cdot A(\theta) \\
C V(\theta)=K_{\theta} \cdot F_{\theta}(\theta) \\
K_{\theta}=\max \left\langle c_{d}(\theta) \cdot A(\theta)\right\rangle
\end{gathered}
$$

Normalizando também a função fluxo em $F_{r}($.$) , temos:$

$$
\Psi\left(\frac{p_{E}(t)}{p_{S}(t)}\right)=\frac{1}{\sqrt{2}} \cdot F_{r}\left(\frac{p_{E}(t)}{p_{S}(t)}\right)
$$


Empregando estas normalizações na equação (8):

$$
\begin{gathered}
\dot{m}(t)=K_{\theta} \cdot F_{\theta}(\theta) \cdot \frac{p_{E}(t)}{\sqrt{R \cdot \vartheta_{E}(t)}} \cdot \frac{1}{\sqrt{2}} \cdot F_{r}\left(\frac{p_{E}(t)}{p_{S}(t)}\right) \\
\dot{m}(t)=f\left(p_{E}, v_{E}\right) \cdot F_{\theta}(\theta) \cdot F_{r}\left(\frac{p_{E}(t)}{p_{S}(t)}\right)
\end{gathered}
$$

As funções $F_{\theta}(\theta)$ e $F_{r}($.$) devem ser identificadas experimentalmente e$ armazenadas no hardware de prototipagem, pois estas funções não variam com o ponto de operação. A função $f\left(p_{E}, \mathrm{v}_{E}\right)$ é da seguinte forma, com $K_{C O R R}$ constante:

$$
f\left(p_{E}, \mathrm{v}_{E}\right)=K_{\text {CORR }} \cdot \frac{p_{E}(t)}{\sqrt{v_{E}(t)}}
$$

De forma a integrar a pressão no modelo do coletor, podemos usar a equação (17) conjuntamente com a equação (3) para a válvula borboleta ou válvula de marcha lenta, devido nesta válvula o fluxo gasoso ter temperatura próxima à temperatura ambiente. Para a válvula de recirculação, caso se situe no duto de recirculação antes da refrigeração, teremos variância significativa da temperatura do fluxo conforme o ponto de operação. Logo, para este caso, recomenda-se o uso das equações (8) e (9) conjuntamente com a equação (1).

Caso o leitor obtiver em (17) gradiente infinito em $F_{r}(1)$ ou em (9) gradiente infinito em $\Psi(1)$, ao realizar a integral dita no parágrafo anterior haverá um problema matemático, pois violará o critério de Lipschitz (vide [5]).

Para contornar este problema, podemos adotar um valor limite para a função fluxo até um valor de razão de pressão próximo a 1 e, depois desse valor, considerar uma outra função que é contínua à primeira e oferece uma transição suave no ponto limite e que não possui gradiente infinito quando a razão de pressão é igual a 1. Em outras palavras, adota-se um limite $\Pi_{\text {LIMITE }}=\left|\frac{p_{S}}{p_{E}}\right|_{\text {LIMITE }}<1$ e, para razões $\Pi \geq$ $\Pi_{\text {LIMITE }}$, usa-se a seguinte função fluxo [1]: 


$$
\begin{gathered}
\Psi(\Pi)=a \cdot(\Pi-1)^{3}+b \cdot(\Pi-1) \\
a=\frac{\Psi_{\text {LIMITE}} \cdot\left(\Pi_{\text {LIMITE }}-1\right)-\Psi_{\text {LIMITE }}}{2 \cdot\left(\Pi_{\text {LIMITE }}-1\right)^{3}} \\
b=\Psi_{\text {LIMITE }}-3 \cdot a \cdot\left(\Pi_{\text {LIMITE }}-1\right)^{2}
\end{gathered}
$$

Sendo $\Psi_{\text {LIMITE }}$ o valor de $\Psi($.$) no limite \Pi_{\text {LIMITE }}$, e $\Psi_{\text {LIMITE }}$ o valor da derivada de $\Psi($. no limite $\Pi_{\text {LIMITE }}$.

\section{Modelagem do fluxo de massa através de um cilindro}

De forma a equacionar o fluxo mássico através de um cilindro, modelamos o motor como uma bomba de ar. Para uma bomba de ar ideal, tem-se [6]:

$$
\dot{m}(t)=\rho \cdot \dot{V}(t)
$$

Corrigimos a fórmula usando um termo nomeado eficiência volumétrica, que computa o distanciamento do motor de uma bomba de gás ideal [6]:

$$
\dot{m}(t)=\lambda_{\mathrm{L}} \cdot \rho \cdot \dot{V}(t)
$$

Aonde,

$\dot{m}(t)$ : Fluxo de gás através da bomba; $\lambda_{\mathrm{l}}$ : Eficiência volumétrica; $\rho$ : Densidade dos gases no duto de entrada; $\dot{V}(t)$ : Derivada temporal do volume da câmara de admissão de gás.

Como a admissão de ar ocorre apenas no ciclo de admissão, tem-se que o valor médio do fluxo é dado por [1]:

$$
\dot{m}(t)=\rho \cdot \lambda_{\iota} \cdot \frac{V_{d}}{N} \cdot \frac{\omega_{e}(t)}{2 \cdot \pi}
$$


Aonde,

$V_{d}$ : Volume de descolamento do motor; $N$ : Metade do número de tempos do motor. Para motor de dois tempos, $N=1$. Para motor de quatro tempos, $N=2 ; \omega_{e}(t)$ : Velocidade do motor.

Para analisar como a composição variada gasolina/etanol interfere na eficiência volumétrica, far-se-á algumas análises:

Ao se vaporizarem no duto de entrada, os vapores de combustível diminuem a temperatura da mistura gasosa circundante no duto, aumentando a eficiência volumétrica [7]. Existem duas propriedades importantes nesta conjuntura: a entalpia de vaporização e a volatilidade da mistura de combustível. A volatilidade tenderia a influir em maior resfriamento da mistura gasosa circundante, devido aos vapores de combustível passarem mais tempo nos dutos. Também exerceria a mesma influência maiores entalpias de vaporização, pois proporciona maior absorção de energia por unidade de massa evaporada. No caso do etanol, o valor elevado de entalpia de vaporização sobressai-se a sua baixa volatilidade, no que concerne à diminuição da temperatura de gases admitidos pelo cilindro [8].

Todavia, há um aspecto que influi fortemente este panorama: boa parte do calor de vaporização da mistura de combustível presente no filme não é retirado da mistura gasosa circundante, mas das superfícies dos dutos e das válvulas de entrada, de forma que a variação da temperatura da mistura gasosa circundante é desprezível conforme se varia a composição gasolina/etanol [9].

Thomas Coppin em [10] formulou a eficiência volumétrica para composição variada gasolina/etanol usando um índice $\xi$, que é a fração de combustível vaporizado que retirou o calor da mistura gasosa circundante. Segundo esta formulação, para $\xi<$ 0.25 a eficiência volumétrica sempre diminui com adição de etanol à gasolina.

Resultados experimentais demonstram que, de fato, há queda na eficiência volumétrica ao adicionar etanol na gasolina. Entretanto, no uso de E100 (situação mais crítica), constatou-se que para determinado motor de estudo a diferença na 
eficiência volumétrica com relação à gasolina é sempre menor que $5 \%$ independente das condições de operação [11]. Em outro estudo, para E85, constatou-se que esta diferença é sempre menor que $4 \%$ para outro motor de estudo [10]. Portanto, recomenda-se desprezar a composição variada gasolina/etanol na formulação da eficiência volumétrica.

A eficiência volumétrica depende de uma série de fatores. O leitor poderá encontrar na literatura várias formas de se modelar a eficiência volumétrica, justamente por que cada autor escolhe aqueles fatores que julga serem mais importantes. Neste artigo, exporemos a modelagem da eficiência volumétrica presente em [1]. Nesta referência, equaciona-se a eficiência volumétrica apenas em função da geometria do motor, da carga (pressão no coletor de admissão) e da velocidade do motor:

$$
\begin{aligned}
& \lambda_{\mathrm{l}}\left(p_{a d m}, \omega_{e}\right)=\lambda_{\mathrm{t} p}\left(p_{a d m}\right) \cdot \lambda_{\mathrm{t} \omega}\left(\omega_{e}\right) \\
& \lambda_{\mathrm{\iota p}}\left(p_{m}\right)=\frac{V_{c}+V_{d}}{V_{d}}-\left(\frac{p_{\text {exh }}}{p_{\text {adm }}}\right)^{1 / \kappa} \cdot \frac{V_{c}}{V_{d}}
\end{aligned}
$$

Aonde,

$V_{c}$ : Volume de compressão; $V_{d}$ : Volume de deslocamento; $p_{\text {exh }}$ : Pressão no coletor de escape no centro de exaustão (do inglês: exhaust center); $p_{a d m}$ : Pressão no coletor de admissão no centro de admissão (do inglês: intake center).

A equação (25) trata-se de uma aproximação, pois há uma simplificação em assumir fatores distintos para a influência de $p_{a d m}$ e $\omega_{e}$ na eficiência volumétrica.

O ganho em se usar as equações (25) e (26) conjuntamente é a facilidade para se identificar a eficiência volumétrica, pois desta forma apenas é necessário identificar $\lambda_{\mathrm{l} \omega}\left(\omega_{e}\right)$.

A identificação da eficiência volumétrica pode ser feita depois de modelado, a partir das seções 1 e 2, o fluxo de saída do coletor de admissão. Portanto, adota-se 
$\dot{m}(t)$ como conhecido na equação (24), o que permite obter experimentalmente a eficiência volumétrica.

\section{CONCLUSÃO}

Os modelos de admissão de ar foram apresentados e mostrou-se como eles podem ser usados para composição variada gasolina/etanol. Expõe-se que as estruturas do modelo permanecem as mesmas dos modelos para gasolina, devido às propriedades de interesse para estes modelos permanecerem próximas do valor original mesmo com adição de etanol. Dentre estas propriedades, podemos citar a capacidade calorífica molar média e a massa molar dos gases queimados e a eficiência volumétrica.

\section{REFERÊNCIAS}

[1] GUZZELLA, Lino; ONDER, Christopher. Introduction to modeling and control of internal combustion engine systems. 2. ed. Springer Science \& Business Media, 2010.

[2] AGARWAL, Deepak; SINGH, Shrawan Kumar; AGARWAL, Avinash Kumar. Effect of Exhaust Gas Recirculation (EGR) on performance, emissions, deposits and durability of a constant speed compression ignition engine. Applied Energy, v. 88, n. 8, p. 2900-2907, 2011.

[3] ISERMANN, Rolf; Engine Modeling and Control; Springer. First Edition. 637p. 2014.

[4] MOSKWA, John Joseph. Automotive engine modeling for real time control. Tese de Doutorado. Massachusetts Institute of Technology. 1988. 
[5] VIDYASAGAR, M. Nonlinear systems analysis. 2nd ed. Philadelphia, Society for Industrial and Applied Mathematics. 498 p. Classics in applied mathematics. 2002.

[6] BARAN, Nicolae; ZAID, Abulghanam; BESNEA, Daniel; DUMINICA, Despina; Theoretical and experimental research of a new type of rotating volumetric pump; The Romanian Review Precision Mechanics, Optics \& Mechatronics, No. 29. 2006.

[7] ANDERSSON, P.; ERIKSSON, L.; Cylinder Air Charge Estimator in Turbocharged SI-Engines. SAE Technical Paper, 2004.

[8] OWEN, K.; COLEY, Trevor; WEAVER, Christopher S.; OWEN, K. Automotive fuels reference book. 2nd ed. Warrendale, PA, Society of Automotive Engineers. 963 p. 1995.

[9] DE SIMIO, Luigi; GAMBINO, Michele; IANNACCONE, Sabato. Effect of ethanol content on thermal efficiency of a spark-ignition light-duty engine. ISRN Renewable Energy. Volume 2012. Article ID 219703.

[10] COPPIN, Thomas. Analyse et modélisation des moteurs Flexfuel pour leur contrôle. Tese de Doutorado. École nationale supérieure d'ingénieurs (Poitiers). 2012.

[11] ENGLER-PINTO, C.; NADAI, L. de; Volumetric Efficiency and Air-Fuel Ratio Analysis For Flex Fuel Engines. SAE Technical Paper. 2008. 